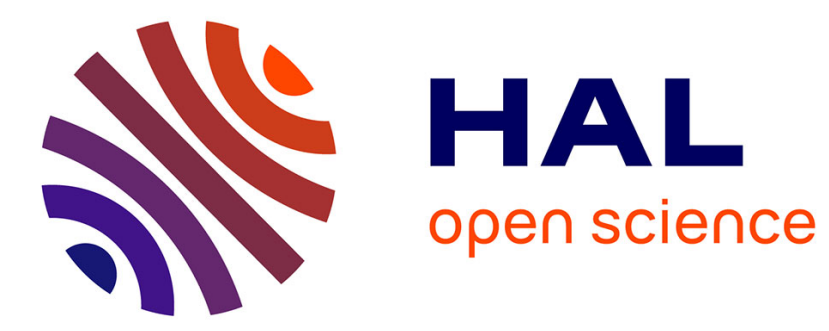

\title{
Polarimetric analysis of stress anisotropy in nanomechanical silicon nitride resonators
}

\author{
T. Capelle, Y. Tsaturyan, A. Barg, A. Schliesser
}

\section{To cite this version:}

T. Capelle, Y. Tsaturyan, A. Barg, A. Schliesser. Polarimetric analysis of stress anisotropy in nanomechanical silicon nitride resonators. Applied Physics Letters, 2017, 110 (18), pp.181106. 10.1063/1.4982876 . hal-03470029

\section{HAL Id: hal-03470029 \\ https://hal.science/hal-03470029}

Submitted on 8 Dec 2021

HAL is a multi-disciplinary open access archive for the deposit and dissemination of scientific research documents, whether they are published or not. The documents may come from teaching and research institutions in France or abroad, or from public or private research centers.
L'archive ouverte pluridisciplinaire HAL, est destinée au dépôt et à la diffusion de documents scientifiques de niveau recherche, publiés ou non, émanant des établissements d'enseignement et de recherche français ou étrangers, des laboratoires publics ou privés. 


\section{Polarimetric analysis of stress anisotropy in nanomechanical silicon nitride resonators ${ }^{(i)}$}

Cite as: Appl. Phys. Lett. 110, 181106 (2017); https://doi.org/10.1063/1.4982876

Submitted: 07 February 2017 • Accepted: 20 April 2017 • Published Online: 01 May 2017

T. Capelle, (D) Y. Tsaturyan, A. Barg, et al.

\section{COLLECTIONS}

EP This paper was selected as an Editor's Pick
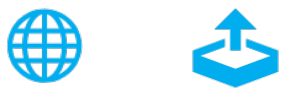

\section{ARTICLES YOU MAY BE INTERESTED IN}

High quality mechanical and optical properties of commercial silicon nitride membranes

Applied Physics Letters 92, 103125 (2008); https://doi.org/10.1063/1.2884191

A phononic bandgap shield for high-Q membrane microresonators

Applied Physics Letters 104, 023510 (2014); https://doi.org/10.1063/1.4862031

Optimized optomechanical crystal cavity with acoustic radiation shield

Applied Physics Letters 101, 081115 (2012); https://doi.org/10.1063/1.4747726

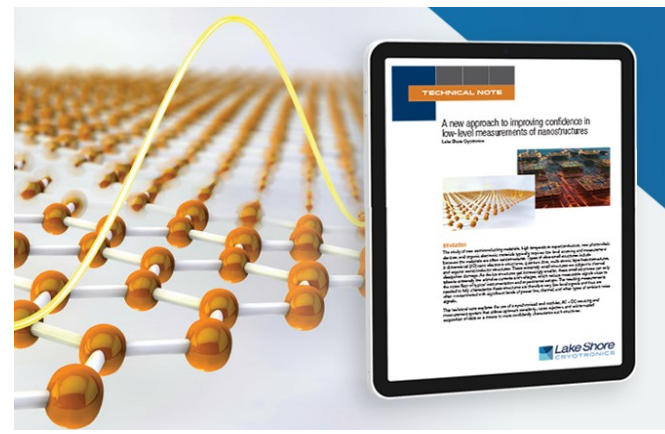

\section{A new approach to low-level} measurements of nanostructures

Read our technical note

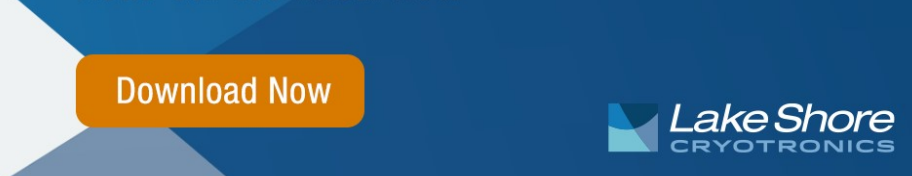




\title{
Polarimetric analysis of stress anisotropy in nanomechanical silicon nitride resonators
}

\author{
T. Capelle, ${ }^{\text {a) }}$ Y. Tsaturyan, A. Barg, and A. Schliesser ${ }^{\text {b) }}$ \\ Niels Bohr Institute, Blegdamsvej 17, 2100 Copenhagen, Denmark
}

(Received 7 February 2017; accepted 20 April 2017; published online 1 May 2017)

\begin{abstract}
We realise a circular gray-field polariscope to image stress-induced birefringence in thin (submicron thick) silicon nitride membranes and strings. This enables quantitative mapping of the orientation of principal stresses and stress anisotropy, complementary to, and in agreement with, finite element modeling. Furthermore, using a sample with a well-known stress anisotropy, we extract a value for the photoelastic (Brewster) coefficient of silicon nitride, $C \approx(3.4 \pm 0.1) \times 10^{-6} \mathrm{MPa}^{-1}$. We explore possible applications of the method to analyse and quality-control stressed membranes with phononic crystal patterns. Published by AIP Publishing.
\end{abstract}

[http://dx.doi.org/10.1063/1.4982876]

Silicon nitride membranes and strings under high tensile stress have excellent mechanical and optical properties, ${ }^{1,2}$ making them a widely used platform to study the behaviour of mechanical systems in the quantum regime. ${ }^{3-10}$ Recently, further enhancement of these properties through in-plane patterning has been explored. Examples include one- ${ }^{11}$ and twodimensional ${ }^{12-14}$ subwavelength optical grating and photonic crystal structures which can boost the reflectivity of SiN beyond $99.9 \%$. In the mechanical domain, "trampoline" resonators ${ }^{15}$ combine thin tethers with a light central pad to achieve low-mass, low-frequency resonators with high quality factors. ${ }^{13,16,17}$ Patterning with phononic bandgap structures ${ }^{18-20}$ can suppress radiation losses and, if combined with optimised dissipation dilution through "soft clamping,",20 yield extreme quality factors and room-temperature $Q \times f$ products beyond $10^{14} \mathrm{~Hz}$.

In all instances of patterning, the stress relaxes to a new equilibrium distribution according to the pattern boundary conditions. This changes dramatically the mechanical, and potentially, via photoelastic coupling, optical properties of the structure. In the absence of a laboratory diagnostic instrument, it has so far been necessary to rely on finite element modeling (FEM) to simulate the stress redistribution. In addition, little is known about the photoelastic coupling in silicon nitride. ${ }^{21,22}$ To address these deficiencies, we have realised a highly sensitive polarimetric setup which allows quantitative imaging of stress-induced birefringence.

Among the numerous possibilities to implement an imaging polarimeter (or polariscope), ${ }^{23}$ we have chosen to build a circular gray-field polariscope. Its basic idea is to illuminate the sample with circularly polarised light and analyse the ellipticity of the transmitted beam's polarisation in a spatially resolved manner. This approach has a decisive advantage over plane polariscopes working with linear polarisation when it comes to measuring small optical retardation $\delta$ : as we will demonstrate, in the circular polariscope, the optical signal is $\propto \delta$, whereas in the plane polariscope, it is only $\propto \delta^{2}$. In our setup (Fig. 1 and Table I), we use a light emitting diode (Thorlabs M780LP1) as a light source, followed by a bandpass filter that eases the requirements on achromaticity of the subsequent optical elements. The source is imaged on a diffuser which, together with an aspheric condenser lens, provides a Köhler-like illumination of the sample. Before reaching the sample, the circular polarisation state is defined by a high-contrast polariser and a quarterwave plate $(\lambda / 4)$. After the sample, a motor-controlled rotating half-waveplate and a polarising beam splitter cube (PBS) analyse the polarisation state. Rotating the waveplate was found to yield better results than rotating various kinds of polarisers, which tended to displace the beam and thus create image artifacts. A microscope objective (Achrovid $5 \times$ ) and a plano-convex lens image part of the sample on a CCD camera (Mightex CGE B013-U) with a magnification of 10. To avoid having the microscope objective before the half waveplate and the PBS, which could have yielded extra (and inhomogeneous) retardation, we used a long working distance microscope ( $37 \mathrm{~mm}$ ) and a $400 \mathrm{~mm}$ focal length lens to obtain the desired magnification.

It is straightforward to compute the expected signal via Jones calculus. Each area element of the sample can be treated as a general retarder described by the Jones matrix

$$
\mathbf{S}_{\delta, \theta}=\mathbf{R}_{\theta}^{-1}\left(\begin{array}{cc}
e^{-i \delta / 2} & 0 \\
0 & e^{+i \delta / 2}
\end{array}\right) \mathbf{R}_{\theta},
$$

where $\theta$ is the azimuthal angle of the polarisation eigenstate basis with respect to a fixed laboratory reference, $\delta$ is the retardation phase, and

$$
\mathbf{R}_{\theta}=\left(\begin{array}{cc}
\cos (\theta) & -\sin (\theta) \\
\sin (\theta) & \cos (\theta)
\end{array}\right),
$$

is the canonical rotation matrix. Specifically, in the case of stress birefringence of a SiN membrane, $\theta$ denotes the direction of the principal stress in the membrane plane, and $\delta$ is the retardation induced by stress anisotropy

$$
\delta=\frac{2 \pi}{\lambda} C d \Delta \sigma
$$

\footnotetext{
a) Also at Laboratoire Kastler Brossel, Paris, France.
}

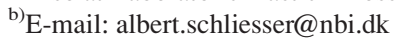




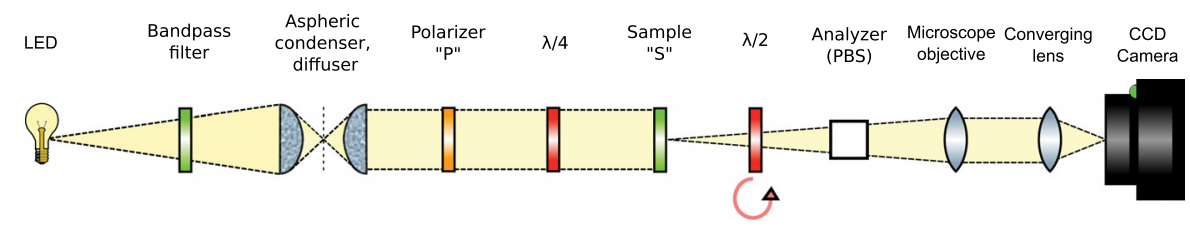

FIG. 1. Gray field polariscope setup. A stepper motor rotates the half wave plate. See text for more details.

where $\lambda$ is the wavelength of the light source, $\Delta \sigma$ the stress anisotropy, $d$ the thickness of the sample, and $C$ the Brewster coefficient, which is a material constant. The other waveplates can easily be represented by similar matrices, setting $\delta=\pi / 2$ for quarter waveplates and $\delta=\pi$ for half waveplates. The analyzer at the end projects the polarisation state, as described by the matrix

$$
\mathbf{P}_{0}=\left(\begin{array}{ll}
1 & 0 \\
0 & 0
\end{array}\right)
$$

If we introduce $\alpha$ as the angle of the output half waveplate with respect to the output polariser, the intensity at each camera pixel is given by $I_{\text {gray }} \propto \vec{J}_{\text {out }}^{\dagger} \cdot \vec{J}_{\text {out }}$, with

$$
\vec{J}_{\text {out }}=\mathbf{P}_{0} \mathbf{S}_{\pi, \alpha} \mathbf{S}_{\delta, \theta} \vec{J}_{\text {in }}
$$

assuming, for simplicity, a perfect input polarisation $\vec{J}_{\text {in }}=(1,-i)^{T} / \sqrt{2}$.

We denote the Fourier transform of the output signal with respect to time $t$ as $\tilde{I}_{\text {gray }}$, during which the half-wave plate rotates with constant angular velocity $\omega \equiv \frac{\partial \alpha}{\partial t}$. Then, it is easy to show that

$$
\frac{\tilde{I}_{\text {gray }}(4 \omega)}{\tilde{I}_{\text {gray }}(0)}=\frac{1}{2} i e^{-2 i \theta} \sin (\delta) .
$$

We can thus extract the entities of interest by calculating

$$
\delta=\arcsin \left(2\left|\frac{\tilde{I}_{\text {gray }}(4 \omega)}{\tilde{I}_{\text {gray }}(0)}\right|\right)
$$

and

$$
\theta=-\frac{1}{2} \arg \left(\frac{\tilde{I}_{\text {gray }}(4 \omega)}{\tilde{I}_{\text {gray }}(0)}\right)+\theta_{0}
$$

Here, $\theta_{0}$ is an offset angle, given by the angle of the first polariser plus $\pi / 4$, which corresponds to the position of the quarter waveplate. Indeed, there is no phase shift when the axis of the quarter waveplate is aligned with the principal axis of the sample.

The data are acquired and processed as follows: images are taken at 10 angular positions for one full rotation.

TABLE I. Technical characteristics of the polarimetric setup.

\begin{tabular}{lc}
\hline \hline LED central wavelength $(\mathrm{nm})$ & 780 \\
Bandpass filter bandwidth $(\mathrm{nm})$ & 10 \\
Working distance of the microscope objective $(\mathrm{mm})$ & 37 \\
Focal length of the imaging lens $(\mathrm{mm})$ & 400 \\
Pixel size $(\mu \mathrm{m})$ & $3.75 \times 3.75$ \\
Resolution of the CCD camera & $1280 \times 960$ \\
Bit depth of the CCD camera ADC & 12 \\
\hline
\end{tabular}

At each position, we acquire a large number of frames (typically 150), from which a frame acquired with the shutter closed is subtracted. This is done in order to remove the dark current, which corresponds to an offset due to the thermal electrons detected using the CCD camera. The procedure is repeated without the sample to acquire background images. A typical raw dataset can be found in Figure 2(b). The main oscillation seen here is due to a slight ellipticity in the input optical field, whereas the actual signal oscillates twice as fast but is significantly smaller. The background image features also a higher mean value, as the sample reflects a small part of the input beam. For each image, treated as a twodimensional matrix, we calculate the normalized complex quantity $\tilde{I}_{\text {gray }}(4 \omega) / \tilde{I}_{\text {gray }}(0)$, which takes into account the inhomogeneous illumination of the sample. Finally, the background is subtracted in the Fourier space from the measurements involving the sample. This yields a complex matrix, which is translated into the orientation $\theta$ and retardation $\delta$ following Eqs. (7) and (8).

For initial validation, we performed a measurement on a sample with a particularly simple geometry, namely, a $210 \mathrm{~nm}$ thick silicon nitride ribbon. We used standard nanofabrication techniques to realise this sample, starting with

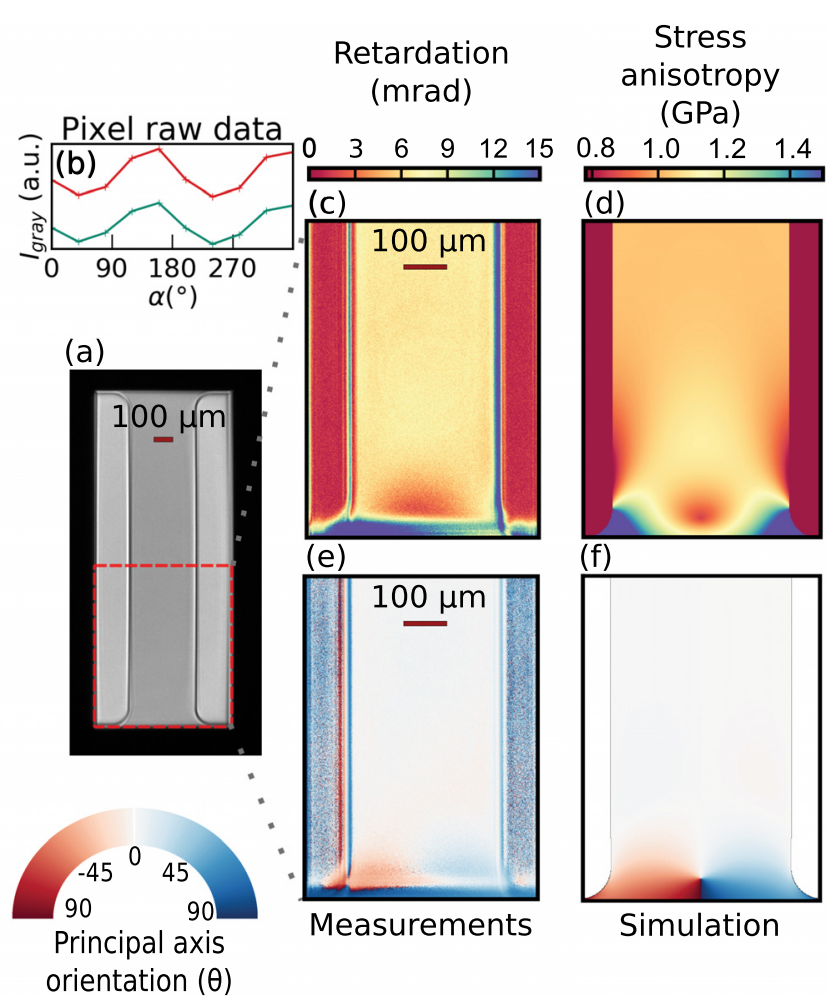

FIG. 2. Analysis of a SiN ribbon. (a) CCD photograph of the sample; the ribbon is show in dark gray. (b) Raw signal for a pixel in the center of the ribbon. The Red curve corresponds to the background data, and the green curve corresponds to the actual data. (c) Measured retardation $\delta$. (d) Simulated stress anisotropy $\Delta \sigma$. (e) Measured angle $\theta$ of the optical axis. (f) Simulated angle of principal stress. 
low-pressure chemical vapor deposition (LPCVD) of $210 \mathrm{~nm}$ stoichiometric silicon nitride on a $500 \mu \mathrm{m}$ double-side polished silicon wafer. The chosen deposition parameters create a film with an isotropic tensile stress of ca. 1.2 GPa. Subsequently, ribbons are defined by UV photolithography and reactive ion etching. The photoresist is removed, and the wafer is stripped of its native silicon oxide layer by a buffered hydrofluoric acid (BHF) dip. Finally, the ribbons are released using an anisotropic $\mathrm{KOH}$ etch from the wafer's backside. Since the ribbons are clamped only from two sides, the stress in the free direction relaxes close to zero.

Figure 2 shows the measured stress birefringence of such ribbons as obtained using our method. The results agree well with our physical intuition, as well as finite-element modelling (COMSOL Multiphysics): at the center of the ribbon, the stress is large along the direction of the ribbon and nonexistent in the orthogonal direction, and so, the anisotropy is maximal. Closer to the clamping region, the ribbon also gets stressed in the orthogonal direction, due to the proximity to the silicon support, reducing the anisotropy. The fillets in the ribbon's corners are expected to display large stress anisotropy (Fig. 2(d)). However, this could not be recovered in our setup, possibly due to diffraction artifacts (see below). The orientation $\theta$ of the principal stress is also reproduced correctly: homogeneously, along the ribbon $\left(\theta \approx 0^{\circ}\right)$ in its center, while close to the clamp, it rotates by $\pm 90^{\circ}$, preserving the horizontal (left/right) symmetry of the geometry, cf. Fig. 2(e).

The good agreement prompts us to apply this approach to measure the Brewster coefficient, relating stress anisotropy to birefringence, of $\mathrm{SiN}$, for which quantitative data are scarce. ${ }^{21,22}$ To that end, we measured a second ribbon, whose initial stress was carefully determined to be $\sigma=1190 \pm 20$ MPa before release of the ribbon. ${ }^{24}$ In order to take into account the change in the boundary conditions during the release of the ribbon, we have to correct this value by a factor $1-\nu$, where $\nu$ is the Poisson ratio of the material, assumed here to be equal to 0.27 . The result is presented in Figure 3 . We obtain a retardation of $6.4 \pm 0.2 \mathrm{mrad}$ by averaging a $\sim 170 \times 260 \mu \mathrm{m}^{2}$-area in the central region of the ribbon.

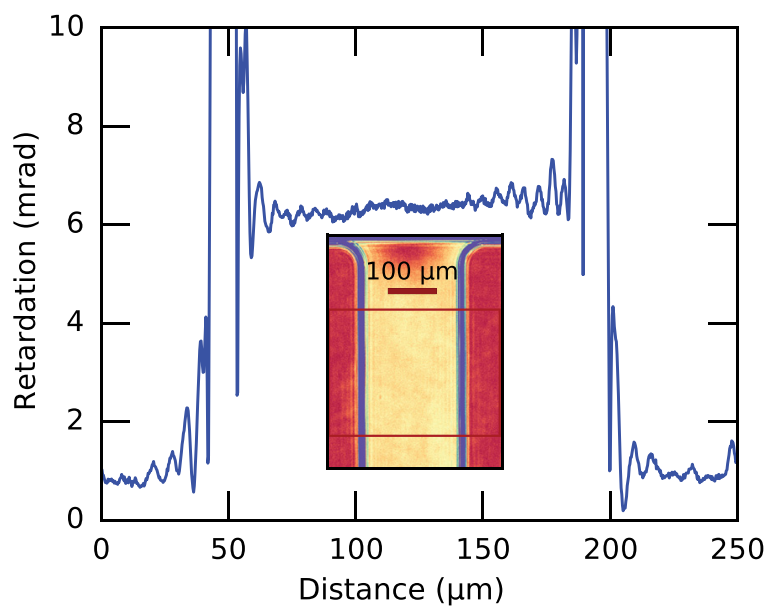

FIG. 3. Retardation measurement on a sample with calibrated stress. Shown is an average over all horizontal line cuts between the two red lines shown in the inset, where stress is approximately homogeneous. The inset shows a full retardation image.
For a quantitative comparison, we correct this value for the multiple reflections inside the film, which are not entirely negligible due to the relatively high $(n \approx 2.0)$ refractive index of SiN. To do so, we used a transfer matrix model for the complex transmittance of this sample, ${ }^{25}$ expanding it to the first order in a small variation of the refractive index. This yielded a correction parameter of $\eta=1.26$ for the effective thickness of the sample. The Brewster coefficient of SiN can then be evaluated as

$$
C=\frac{\delta}{2 \pi} \frac{\lambda}{\eta d} \frac{1}{\sigma(1-\nu)} \approx(3.4 \pm 0.1) \times 10^{-6} \mathrm{MPa}^{-1},
$$

where $d=210 \mathrm{~nm}$ is the sample thickness. Remarkably, this is two orders of magnitude lower than the value proposed by Campillo and $\mathrm{Hsu}^{26}$ but close to the value of silica $(C \approx 4$ $\left.\times 10^{-6} \mathrm{MPa}^{-1}\right),{ }^{27}$ another amorphous transparent dielectric. This mistake arises from the fact that this previous work measured the refractive index and the stress for different $\mathrm{SiH}_{2} \mathrm{Cl}_{2}: \mathrm{NH}_{3}$ gas flow ratios during the LPCVD process and not the variation of the refractive index due to mechanical stress.

With such calibration at hand, we proceed to applying polarimetric stress analysis to more complicated resonator structures. Membrane resonators with a phononic bandgap shield are of particular interest. In the context of silicon nitride membranes, it has been shown that phononic crystal structures can suppress the transmission of vibrations, ${ }^{28,29}$ resulting in the suppression of dissipation through phonon tunneling, ${ }^{29,30}$ whose avoidance had previously required delicate and often unreliable clamping techniques. ${ }^{2}$ Patterning a phononic crystal structure directly onto the membrane not only suppresses phonon tunneling losses but also enhances the dilution of internal losses dramatically, enabling an increase in the quality factor by more than an order of magnitude ${ }^{20}$ as compared to conventional membrane resonators embedded in silicon phononic crystal structures. Stress analysis by polarimetry in such complex geometries can be a reliable and simple tool to assess the periodicity of stress anisotropy as required for the formation of a bandgap.

To demonstrate this potential, we realised membranes ( $d \approx 210 \mathrm{~nm}$ ) with a honeycomb pattern of $\sim 200 \mu \mathrm{m}$-diameter holes, fabricated using the same techniques as for the ribbons. Such a patterned membrane exhibits a bandgap as previously described. ${ }^{20}$ Once again, we performed a measurement of the retardation, as described above, with the result shown in Figure 4. As expected, we observe an enhanced stress anisotropy in the tethers (i.e., the narrow regions between the circular holes) (Fig. 4(a)). In particular, a cut along a tether reveals a peak, symmetric with respect to the center of the tether, and a maximum stress anisotropy of $\Delta \sigma \sim 2.3 \mathrm{GPa}$. Good agreement with the predicted stress anisotropy in this region confirms the previously computed value of the Brewster coefficient (Fig. 4(b)).

Near the edges of the membrane, large retardation values ( $\gtrsim 15 \mathrm{mrad}$ ) appear as dark blue rings around the holes (Fig. 4(a)). The observation of these features already in single-shot images rules out eccentric mechanical rotation as their cause. Rather, we attribute them to diffraction effects. This is supported by the presence of several weak concentric 

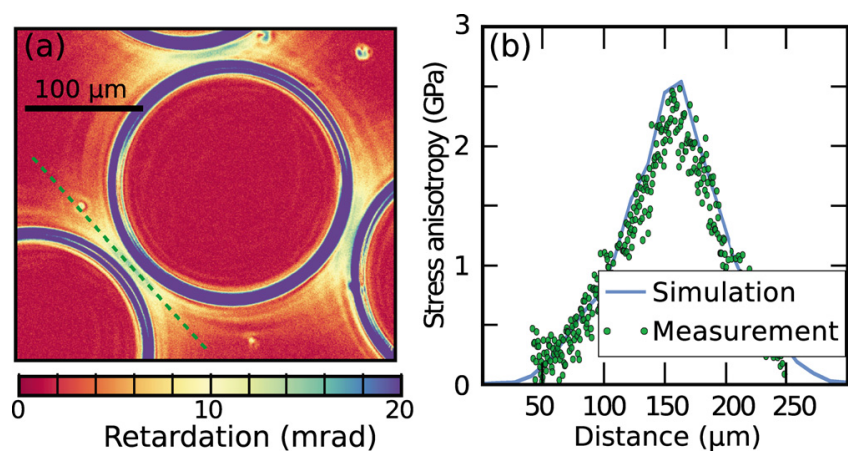

FIG. 4. Analysis of a patterned membrane. (a) Retardation image. (b) Measured retardation along the dashed line in (a), following a tether between two holes. Values are converted to stress anisotropy via the previously extracted Brewster coefficient and corrected for multiple reflections in the film.

rings around the holes due to higher diffraction orders and by the fact that their length scale is that of the $\sim 4 \mu \mathrm{m}$-Airy disk corresponding to the numerical aperture $(0.12)$ and the wavelength $(780 \mathrm{~nm})$ used. Indeed, diffraction fringes are expected when imaging a transparent film that induces an optical phase shift ${ }^{31}$ —with a sensitive (wavelength-scale) dependence on focusing conditions that we have experimentally observed. The diffraction fringes' precise location (and phase) depends on the optical thickness of the SiN film. Since the effective thickness varies as we vary the analysed polarization, this generates pixel intensity modulation that our analysis algorithm mistakes for birefringence also outside the actual membrane.

In spite of such artifacts, it is straightforward to recognise defective membranes. Figure 5 shows retardation images of an undamaged membrane and one with one or several broken tethers, as a comparison. The ensuing local stress release leaves a clear signature in the membrane pads, in the form of yellow bands that break the phononic crystal symmetry. This allows the identification of a defective membrane without the need for a more involved bandgap measurement. One could envision using this approach in assessing the overall performance of the device (e.g., the quality factor), by comparing
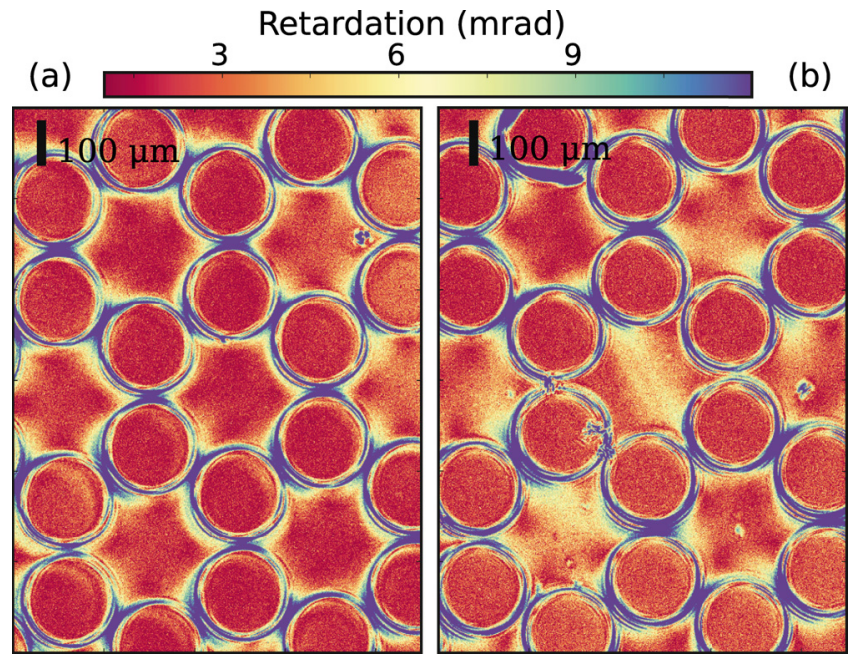

FIG. 5. Comparison between an undamaged membrane (a) and one with a broken tether (b). Stress released in (b) and the broken periodicity can be clearly recognised, allowing identification and localisation of the defect. the stress profile of the defective device with simulations of an undamaged membrane resonator.

In conclusion, using $210 \mathrm{~nm}$-thick membrane resonators, our experimental setup achieves a $\sim 0.2 \mathrm{mrad}$ resolution in retardation over a $\sim(200 \mu \mathrm{m})^{2}$ area. Using the measured Brewster coefficient, this corresponds to a resolution in a stress anisotropy of $\sim 80 \mathrm{MPa}$. Thus, we expect the method to deliver relevant results also for ultrathin $(d \lesssim 50 \mathrm{~nm}) \mathrm{mem}-$ branes of smaller dimensions.

As an imaging modality, our basic microscopy setup achieves moderate transverse resolution on the order of $10 \mu \mathrm{m}$, impaired by diffraction artifacts. Better results should be available with higher N.A. optics. In the present form, it is nonetheless practically sufficient to assess the overall periodicity of patterned membrane resonators as needed to form a bandgap. More generally, our gray field polariscope, validated by FEM simulations, has allowed us to perform a measurement of the Brewster coefficient of SiN, correcting an older value found in the literature. ${ }^{26}$ This can be of interest for optomechanical systems relying on photoelastic coupling in this material. ${ }^{21,22}$

This work received funding from the European Research Council (ERC) under the European Union's Horizon 2020 research and innovation programme (Project Q-CEOM, Grant No. 638765), the European Union Seventh Framework Programme (Project iQUOEMS, Grant No. 323924), and the Danish Council for Independent research (Sapere Aude Grant No. 4002-00060).

${ }^{1}$ B. M. Zwickl, W. E. Shanks, M. Jayich, C. Yang, C. B. Jayich, J. D. Thompson, and J. G. E. Harris, Appl. Phys. Lett. 92, 103125 (2008).

${ }^{2}$ D. J. Wilson, C. A. Regal, S. B. Papp, and H. J. Kimble, Phys. Rev. Lett. 103, 207204 (2009).

${ }^{3}$ T. P. Purdy, P.-L. Yu, R. W. Peterson, N. S. Kampel, and C. A. Regal, Phys. Rev. X 3, 031012 (2013).

${ }^{4}$ T. P. Purdy, R. W. Peterson, and C. Regal, Science 339, 801 (2013).

${ }^{5}$ X. Zhou, F. Hocke, A. Schliesser, A. Marx, H. Huebl, R. Gross, and T. J. Kippenberg, Nat. Phys. 9, 179 (2013).

${ }^{6}$ M. Underwood, D. Mason, D. Lee, H. Xu, L. Jiang, A. B. Shkarin, K. Borkje, S. M. Girvin, and J. G. E. Harris, Phys. Rev. A 92, 061801(R) (2015).

${ }^{7}$ D. J. Wilson, V. Sudhir, N. Piro, R. Schilling, A. Ghadimi, and T. J. Kippenberg, Nature 524, 325 (2015).

${ }^{8}$ R. Peterson, T. P. Purdy, N. Kampel, R. Andrews, P.-L. Yu, K. Lehnert, and C. Regal, Phys. Rev. Lett. 116, 063601 (2016).

${ }^{9}$ W. H. P. Nielsen, Y. Tsaturyan, C. B. Møller, E. S. Polzik, and A. Schliesser, Proc. Natl. Acad. Sci. U.S.A. 114, 62 (2016).

${ }^{10}$ A. Noguchi, R. Yamazaki, M. Ataka, H. Fujita, Y. Tabuchi, T. Ishikawa, K. Usami, and Y. Nakamura, New J. Phys. 18, 103036 (2016).

${ }^{11}$ C. Stambaugh, H. Xu, U. Kemiktarak, J. Taylor, and J. Lawall, Ann. Phys. 527, 81 (2015).

${ }^{12}$ X. Chen, C. Chardin, K. Makles, C. Caër, S. Chua, R. Braive, I. RobertPhilip, T. Briant, P.-F. Cohadon, A. Heidmann, T. Jacqmin, and S. Deléglise, Nat.: Light, Sci. Appl. 6, e16190 (2017).

${ }^{13}$ R. Norte, J. P. Moura, and S. Gröblacher, Phys. Rev. Lett. 116, 147202 (2016).

${ }^{14}$ S. Bernard, C. Reinhardt, V. Dumont, Y.-A. Peter, and J. C. Sankey, Opt. Lett. 41, 5624 (2016).

${ }^{15}$ D. Kleckner, B. Pepper, E. Jeffrey, P. Sonin, S. M. Thon, and D. Bouwmeester, Opt. Express 19, 19708 (2011).

${ }^{16}$ C. Reinhardt, T. Müller, A. Bourassa, and J. C. Sankey, Phys. Rev. X 6, 021001 (2016).

${ }^{17}$ M. J. Weaver, B. Pepper, F. Luna, F. M. Buters, H. J. Eerkens, G. Welker, B. Perock, K. Heeck, S. de Man, and D. Bouwmeester, Appl. Phys. Lett. 108, 033501 (2016). 
${ }^{18}$ A. H. Ghadimi, D. J. Wilson, and T. J. Kippenberg, "Radiation and internal loss engineering of high-stress silicon nitride nanobeams," Nano Lett. (published online).

${ }^{19}$ A. Z. Barasheed, T. Müller, and J. C. Sankey, Phys. Rev. A 93, 053811 (2016).

${ }^{20}$ Y. Tsaturyan, A. Barg, E. S. Polzik, and A. Schliesser, e-print arXiv:1603.07200.

${ }^{21}$ K. Børkje and S. M. Girvin, New J. Phys. 14, 085016 (2012).

${ }^{22}$ K. E. Grutter, M. Davanco, and K. Srinivasan, IEEE J. Sel. Top. Quantum Electron. 21, 61 (2015).

${ }^{23}$ A. Ajovalasit, G. Petrucci, and M. Scafidi, Exp. Tech. 39, 11 (2015).

${ }^{24}$ This measurement was conducted on a separate sample with a $238.6-\mathrm{nm}$ thick SiN layer grown in the same conditions as the ribbon. We take the typical deviation of measured stress in $\mathrm{x}$ and $\mathrm{y}$ directions as combined measurement error and film inhomogeneity of $2 \%$.

${ }^{25}$ The transmittance of a membrane of thickness $d$ and refractive index $n$ is given by $t=\frac{2 i n e^{-i k d}}{2 i n \cos (k d n)+\left(1+n^{2}\right) \sin (n k d)}$.

${ }^{26}$ A. L. Campillo and J. W. P. Hsu, J. Appl. Phys. 91, 646 (2002).

${ }^{27}$ R. Priestley, Proc. SPIE 4346, 1300-1305 (2001).

${ }^{28}$ Y. Tsaturyan, A. Barg, A. Simonsen, L. G. Villanueva, S. Schmid, A. Schliesser, and E. S. Polzik, Opt. Express 22, 6810 (2014).

${ }^{29}$ P. L. Yu, K. Cicak, N. S. Kampel, Y. Tsaturyan, T. P. Purdy, R. W. Simmonds, and C. A. Regal, Appl. Phys. Lett. 104, 023510 (2014).

${ }^{30}$ I. Wilson-Rae, Phys. Rev. B - Condens. Matter Mater. Phys. 77, 245418 (2008).

${ }^{31}$ R. Faust, Proc. Phys. Soc. London, Sect. B 64, 105 (1951). 